\title{
Real-Time Rendering of Teeth with No Preprocessing
}

\author{
Larsen, Christian Thode; Frisvad, Jeppe Revall; Jensen, Peter Dahl Ejby; Bærentzen, Jakob Andreas
}

Published in:

Advances in Visual Computing

Link to article, DOI:

10.1007/978-3-642-33191-6_33

Publication date:

2012

Document Version

Early version, also known as pre-print

Link back to DTU Orbit

Citation $(A P A)$ :

Larsen, C. T., Frisvad, J. R., Jensen, P. D. E., \& Bærentzen, J. A. (2012). Real-Time Rendering of Teeth with No Preprocessing. In G. Bebis, R. Boyle, B. Parvin, D. Koracin, F. Charless, W. Sen, C. Min-Hyung, S. Mantler, J. Schulze, D. Acevedo, K. Mueller, \& M. Papka (Eds.), Advances in Visual Computing: Proceedings of ISVC 2012, Part II (pp. 334-345). Springer. Lecture Notes in Computer Science Vol. 7432 https://doi.org/10.1007/978-3-64233191-6 33

\section{General rights}

Copyright and moral rights for the publications made accessible in the public portal are retained by the authors and/or other copyright owners and it is a condition of accessing publications that users recognise and abide by the legal requirements associated with these rights.

- Users may download and print one copy of any publication from the public portal for the purpose of private study or research.

- You may not further distribute the material or use it for any profit-making activity or commercial gain

- You may freely distribute the URL identifying the publication in the public portal 


\title{
Real-Time Rendering of Teeth with No Preprocessing
}

\author{
Christian Thode Larsen ${ }^{1}$, Jeppe Revall Frisvad ${ }^{1}$, \\ Peter Dahl Ejby Jensen ${ }^{2}$, and Jakob Andreas Bærentzen ${ }^{1}$ \\ 1 Technical University of Denmark \\ 2 3Shape A/S, Holmens Kanal 7, 1060 Kbh. K, Denmark
}

\begin{abstract}
We present a technique for real-time rendering of teeth with no need for computational or artistic preprocessing. Teeth constitute a translucent material consisting of several layers; a highly scattering material (dentine) beneath a semitransparent layer (enamel) with a transparent coating (saliva). In this study we examine how light interacts with this multilayered structure. In the past, rendering of teeth has mostly been done using image-based texturing or volumetric scans. We work with surface scans and have therefore developed a simple way of estimating layer thicknesses. We use scattering properties based on measurements reported in the optics literature, and we compare rendered results qualitatively to images of ceramic teeth created by denturists.
\end{abstract}

\section{Introduction}

It is possible with existing scanning and rendering techniques to create highly realistic digital versions of real actors. These digital versions can blend in naturally with real characters in feature films [1]. The main problem with this type of digital (face) acquisition is the cost in artistic preprocessing. In the cited work, this preprocessing takes up three months of an artist's time, just to refine rendering parameters and do compositing. Once acquired, the digital actor can be rendered in photorealistic movie production quality, or, as an alternative, rendered in real-time at a lower but still relatively high level of realism. However, when we impose a real-time constraint, the eye and mouth regions especially become less realistic [2]. Since mouth and eyes are areas of the face that in particular draw attention, realistic real-time rendering of teeth has been identified as an important avenue for future work [2].

In this paper, we aim at realistic real-time rendering of teeth that does not require artistic preprocessing, image-based texturing, or precise knowledge about the internal structure of teeth. We constrain our rendering technique in this way because it is intended for use in the dental industry. New scanners have been developed that enable fast in-clinic dental impression scanning [3]. Such scanners capture the surface geometry of teeth and eliminate the need for difficult and time consuming plaster casts. If rendering of the scanned teeth is realistic, the dentist can talk to customers about options with respect to crowns or bridges shortly after scanning the oral cavity. Thus these new scanners generate

This is the author's version of the work. The original publication is available at www.springerlink.com and it was published in G. Bebis et al. (Eds.): ISVC 2012, Part II, LNCS 7432, pp. 334-345, 2012. (C) Springer-Verlag Berlin Heidelberg 2012 
a demand for a rendering technique for teeth that adheres to the constraints mentioned above.

The appearance model we present for teeth maintains a strong coupling to existing models for subsurface scattering. In particular, we use a model for single scattering in multiple layers by Hanrahan and Krueger [4]. We combine this model with a technique for real-time skin rendering by Hable et al. [5], in order to estimate the multiple scattering component. Finally, we introduce adjustments to better capture the visual traits of the tooth material while keeping frame rates above 20 fps. By following this particular design, the model has maintained a level of generality that should make it useful for rendering any translucent material with a semi-transparent coating (porcelain is another example).

\subsection{Related Work}

Little work has been published on realistic rendering of teeth. The work most closely related to ours is the layered model presented by Shetty and Bailey [6] and by Shetty [7]. However, artistic preprocessing is needed in order to define the distribution of dentine inside the teeth. This means that the model is only suitable for library tooth geometry and therefore not well-suited for the application we have in mind.

Using a CT scanner, it is possible to capture a volumetric description of a set of teeth. Volume visualisation techniques are typically used to render such a scan $[8,9]$. This has the purpose of visualising the different layers of the teeth rather than reproducing their appearance. It is, however, possible to extract the surfaces of the different layers from a CT scan and use them for rendering. Kim and Park [10] and Rhienmora et al. [11] did this to develop systems to be used in training situations for dentists. Their systems include real-time visualisation of teeth, but the authors focus on haptic rendering rather than visual realism, and, apparently, they use the standard OpenGL pipeline. To improve visual quality and render rate, Yau and Hsu [12] use surfel models and surfel rendering for their dental training system. However, they do not include any translucency effects.

We have found no previous work where layer surfaces extracted from a CT scan have been used to enhance the rendered appearance of a tooth. Although we focus on surface scans, the rendering technique we present would also apply to a tooth surface extracted from a CT scan. And precise knowledge about the dentine surface beneath the enamel should lead to a higher degree of realism.

Wang et al. [13,14] have developed a dental training system based on surface scans of teeth. They face the same problem as we do, namely that they have to estimate the layered geometry inside a tooth. As far as we can tell, they do this manually. They use the tooth's layers, not to get translucency effects in the graphical rendering, but to get effects in the haptic rendering and to display different colours for different tissue types as the dentist drills into the tooth. Again, shading seems to be done using the standard OpenGL pipeline.

Another approach to rendering teeth is image-based texturing. The best example of the realism that can be achieved with this approach is probably the teeth of the digital actress Emily [1]. As discussed in the introduction, the artistic 
preprocessing needed for this approach makes it unsuitable for our application. An image-based texturing approach where artistic preprocessing is not needed has been presented by Pighin et al. [15]. Some manual work is still required to specify correspondences between images and a generic face model. It is unlikely that this type of manual work would be acceptable in an in-clinic dental impression scanning context.

Zhang et al. [16] describe a face reconstruction model where a generic skull (including teeth) is automatically morphed to fit a range scanned face. Their model might enable automatic image-based texturing of the teeth in the morphed skull. Unfortunately, the authors leave this investigation for future work.

Image-based texturing has the potential of achieving highly realistic results. The required image data is, however, not always available, as is the case in the application we are working with.

\section{Theory}

Tooth is a complex composite material that consists of several layers. The innermost layer which is optically significant is dentine. This is a highly scattering material. The dentine layer is beneath an enamel layer which is semi-transparent and possibly covered by a transparent saliva coating. Light scattering in these layers is affected by variations in optical properties, and by variations in the thickness of the enamel and dentine layers. In the following, we describe this subsurface scattering and our simple model for estimating layer thicknesses. Then we suggest a real-time rendering technique based on this theory.

\subsection{Subsurface Scattering}

The input needed for rendering a scattering material are the optical properties of the material, that is, the phase function, $p$, the absorption and scattering coefficients, $\sigma_{a}$ and $\sigma_{s}$, and the index of refraction, $\eta$. These determine the appearance of the material. The Fresnel equations for reflection and the laws of reflection and refraction are used to handle scattering at the surface. These require the index of refraction as input. The remaining properties are used for computing subsurface scattering.

It is common to approximate subsurface scattering by a sum of radiance contributions due to $0^{t h}$-order, $1^{s t}$-order and $n^{\text {th }}$-order scattering. These three contributions are determined by finding partial or full solutions to the radiative transfer equation [17], and are commonly referred to as reduced intensity, single scattering, and multiple scattering, respectively. Analytical solutions for these terms only exist in special cases. To find analytical expressions for the reduced intensity and the single scattering terms, we assume that the layers are homogeneous and locally plane-parallel.

The reduced intensity passing through a layer of thickness $t$ (the layer of refractive index $\eta_{2}$ in figure 1 ) is then $[17,4]$

$$
L^{(0)}=F_{t}^{12} F_{t}^{23} e^{-\tau / \mu_{0}} L_{i},
$$




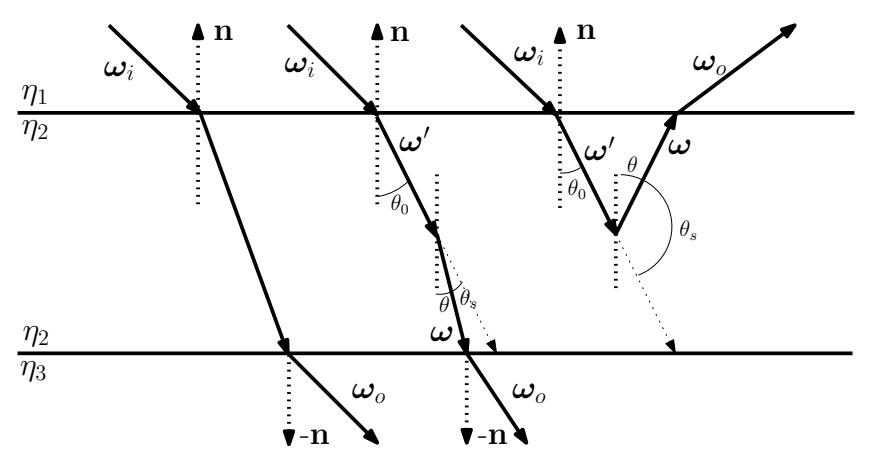

Fig. 1. Illustrating from left to right: reduced intensity, inward single scattering, and outward single scattering.

where $F_{t}^{12}$ and $F_{t}^{23}$ are the Fresnel transmittances at the interfaces between the layers, $\mu_{0}=-\boldsymbol{n} \cdot \boldsymbol{\omega}$ is the cosine of the angle between the inward surface normal and the direction of the refracted light, and, if we let $\sigma_{t}=\sigma_{a}+\sigma_{s}$ denote the extinction coefficient of the layer, $\tau=\sigma_{t} t$ is its optical thickness.

Single scattering is the contribution from light that scatters exactly once inside the medium before it reemerges. If the light emerges at the plane where it was incident, it is called outward scattered light, whereas it is called inward scattered light if it emerges on the opposing plane (figure 1). Both inward and outward single scattering have analytical solutions for constant, collimated light incident on a homogeneous, plane-parallel medium. Under these assumptions, single scattering only depends on the optical thickness of the layer $\tau$ and on the direction of the incident illumination $\boldsymbol{\omega}_{i}$. Inward scattering $(+)$ and outward scattering $(-)$ are then given by $[17,4]$ :

$$
\begin{aligned}
& L_{+}^{(1)}=F_{t}^{12} F_{t}^{23} \alpha p\left(\theta_{s}\right) \frac{\mu_{0}}{\mu_{0}-\mu}\left(e^{-\tau / \mu_{0}}-e^{-\tau / \mu}\right) L_{i}, \\
& L_{-}^{(1)}=F_{t}^{12} F_{t}^{21} \alpha p\left(\theta_{s}\right) \frac{\mu_{0}}{\mu_{0}+\mu}\left(1-e^{-\tau\left(1 / \mu_{0}+1 / \mu\right)}\right) L_{i},
\end{aligned}
$$

where $\alpha=\frac{\sigma_{s}}{\sigma_{t}}$ is the scattering albedo, which describes the probability of scattering versus absorption, and $p$ is the previously mentioned phase function. The law of refraction is used to find the forward direction $\boldsymbol{\omega}^{\prime}$ and the scattering direction $\boldsymbol{\omega}$ inside the medium. These directions lead to the cosine terms $\mu_{0}=-\boldsymbol{n} \cdot \boldsymbol{\omega}^{\prime}$ and $\mu=\boldsymbol{n} \cdot \boldsymbol{\omega}$ and the scattering angle $\theta_{s}=\cos ^{-1}\left(\boldsymbol{\omega}^{\prime} \cdot \boldsymbol{\omega}\right)$. If light scatters back into the forward direction, the cosine terms $\mu$ and $\mu_{0}$ are equal. This results in a division by zero in eq. 2 . In this special case, the following formula should be used instead:

$$
L_{+}^{(1)}=F_{t}^{12} F_{t}^{23} \alpha p\left(\theta_{s}\right) \frac{\tau}{\mu} e^{-\tau / \mu} L_{i}
$$

Assuming a semi-infinite, homogenous medium with a planar surface, it is possible to derive a dipole approximation for the bidirection scattering-surface reflectance distribution function (BSSRDF) using diffusion theory [18]. The dipole 
approximation estimates the fraction of incident radiance which emerges at some distance $r=\left|\boldsymbol{x}_{o}-\boldsymbol{x}_{i}\right|$ from the point of incidence due to multiple scattering. We use the 12-tap method of Hable et al. [5] to estimate the multiple scattering in dentine. Given a surface position $\boldsymbol{x}_{o}$ this method finds the light incident at twelve other surface positions $\boldsymbol{x}_{i}$ in its vicinity (the twelve positions are "tapped"). In practice, this is done in a fragment shader with a sample kernel that consists of twelve weighted pixel positions. These positions define the sampling area around the currently shaded pixel (the kernel origin). The multiple scattering is sampled once for each weighted pixel position by evaluating the BSSRDF and multiplying with the incident radiance that is transmitted through the enamel layer. To get an estimate of the radiance that emerges at the surface of the tooth after multiple scattering in the dentine, the samples are accumulated and attenuated by transmission back through the enamel layer.

\subsection{Optical Properties of Enamel and Dentine}

A study of optics literature has provided us with measured intervals for the absorption and scattering coefficients in enamel and dentine. Table 1 has been constructed from values found in several references [19-22], with emphasis on the work of Fried et al. [21] as it, to our knowledge, presents the most detailed information. In addition, we use a refractive index of 1.33 to model a coating of saliva on the teeth.

Table 1. Approximate optical properties of enamel and dentine at three wavelengths. Scattering and absorption coefficients $\left(\sigma_{a}\right.$ and $\left.\sigma_{s}\right)$ are measured in $\mathrm{cm}^{-1}$.

\begin{tabular}{lcccccc}
\hline material & $\eta$ & $\sigma_{a}$ & $\sigma_{s, 1053 \mathrm{~nm}}$ & $\sigma_{s, 632 \mathrm{~nm}}$ & $\sigma_{s, 543 \mathrm{~nm}}$ & $g$ \\
\hline enamel & 1.63 & $<1$ & $15 \pm 5$ & $60 \pm 18$ & $105 \pm 30$ & $0.96 \pm 0.02$ \\
dentine & 1.49 & $3.5 \pm 0.5$ & $260 \pm 78$ & $280 \pm 84$ & $280 \pm 84$ & $0.93 \pm 0.02$ \\
\hline
\end{tabular}

The approximate mean thickness of the enamel layer is one millimetre (1 $\mathrm{mm})$, while the average distance to the first scattering event $\left(1 / \sigma_{t}\right)$ is around $0.2 \mathrm{~mm}$. Around five scattering events before emergence is too little for the multiple scattering approximation to be accurate. Especially considering that the asymmetry parameter $g$ is close to one, which means that the scattering angle most often is very small. In other words, most light passes through the enamel with very little deviation from the forward direction. We have therefore decided to use only single scattering and reduced intensity for the enamel layer.

Similar analysis reveals that the dentine layer should be approximately one millimetre thick for the multiple scattering approximation to be useful. Since the dentine layer always seems to be a few millimetres thick, we have decided to use the multiple scattering approximation for this material.

Since scattering is highly asymmetric in both enamel and dentine ( $g$ is close to 1 ), we use the Henyey-Greenstein phase function [23] for $p\left(\theta_{s}\right)$ in eqs. $3-4$. 


\subsection{Layer Geometry}

The saliva layer is included in our model using the method of Jensen et al. [24]. This method is purely based on refractive indices and Fresnel's equations, and thus we need not model the thickness of the saliva layer.

To estimate enamel thickness $t$, we model it as varying linearly between top $y_{\max }$ and bottom $y_{\min }$ of the tooth bounding box with height $l_{y}=\left(y_{\max }-y_{\min }\right)$ :

$$
t=t_{\min }+\left(t_{\max }-t_{\min }\right)\left(v_{y}-y_{\min }\right) / l_{y},
$$

where $\boldsymbol{v}=\left(v_{x}, v_{y}, v_{z}\right)$ is a tooth vertex position and $t_{\min }$ and $t_{\max }$ are suitable minimum and maximum enamel thicknesses that are chosen according to the type of tooth (incisor or molar). It is also necessary to choose a reference direction for the interpolation since position of top and bottom depends on whether the tooth is placed in the upper or the lower jaw.

To create the dentine layer, we transform a copy of the tooth surface as follows $\left(\boldsymbol{v} \mapsto \boldsymbol{v}^{\text {new }}\right)$. The geometry is first scaled in the $x z$-plane which is normal to the tooth height axis (the $y$-axis). It is then scaled and offset along the height axis to align the two meshes at the bottom of the bounding box. The scaling vector in the $x z$-plane is determined by

$$
\boldsymbol{s}_{x z}=\left(\left(l_{x}-2 t\right) / l_{x},\left(l_{z}-2 t\right) / l_{z}\right),
$$

where $\boldsymbol{l}=\left(l_{x}, l_{y}, l_{z}\right)$ contains the extend of the bounding box along the three coordinate axes. Tooth vertices are displaced in the $x z$-plane by

$$
\boldsymbol{v}_{x z}^{\text {new }}=\boldsymbol{s}_{x z} *\left(\boldsymbol{v}_{x z}-\boldsymbol{b}_{x z}\right)+\boldsymbol{b}_{x z},
$$

where $\boldsymbol{b}_{x z}$ is the mesh barycentre projected to the $x z$-plane and $*$ denotes element-wise multiplication. To displace the vertices along the $y$-axis, we use

$$
v_{y}^{\text {new }}=y_{\min }+\left(v_{y}-y_{\min }\right)\left(l_{y}-t\right) / l_{y} .
$$

Note that this scaling method is not robust if the concavity of the tooth is high, as the dentine vertices then may become ill positioned.

\subsection{Translucency Near Edges}

The semi-transparency near the edges of teeth is a result of the dentine layer coming to an end. These near-edge regions pose a particular challenge to our method because the remaining enamel is thick enough for more than one scattering event to occur, but too thin for the diffusion approximation. The problem is illustrated in figure 2 .

Our model handles this by sampling the enamel surface with the 12-tap kernel, and subsequently mapping each sample point to a corresponding dentine position. This results in an over-contribution of radiance from the dentine layer, which in turn eliminates the semi-transparency near the edges. To account for this, the dentine-contributed radiance should be corrected by an attenuation factor. We prefer to compute this factor using $a=p_{t} t^{2}$, where $t$ is the thickness of the tooth in the view direction, and $p_{t} \in[0 ; 1]$ controls the level of attenuation. 


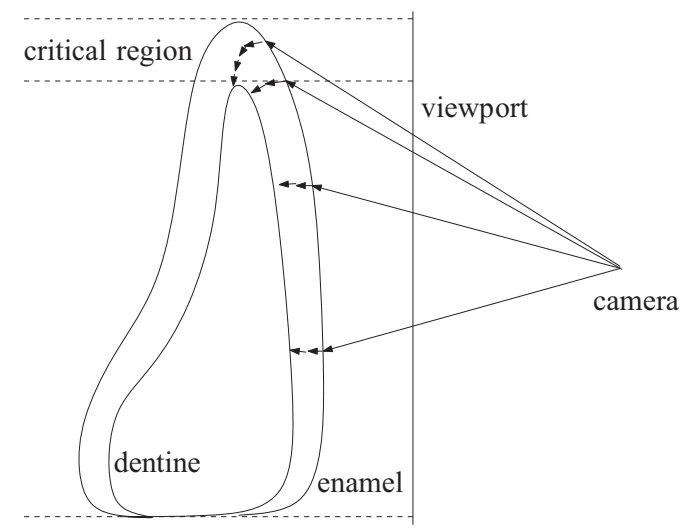

Fig. 2. The near-edge regions of the teeth are critical as they consist entirely of enamel in the view direction. Scattering from the dentine which reaches the edges of the tooth due to a few scattering events is difficult to evaluate in a fragment shader. We use an ad hoc approach to handle this critical region.

\subsection{Tooth Roughness}

While the surface of enamel appears to be relatively smooth, small variations in the surface micro structure may justify some simulation of roughness. A crude yet simple way to introduce roughness is to raise the Fresnel reflectance to an exponent [24]. This roughness value should be chosen entirely based on preference, and more aggressive values will effectively result in a higher amount of transmitted radiance through the layers. In our renderings, we use a Fresnel reflectance exponent of 1.3 for the enamel layer. This has the effect of making the teeth slightly less reflective and slightly whiter. We have not been able to find any physically based measurements of enamel roughness.

\section{Implementation}

The shader implementation combines the covered models and techniques using several vertex shader passes that render information about the geometry of the teeth to textures and a main fragment shader pass that shades the teeth. For each tooth, the solution requires as input the enamel surface geometry, its bounding box and barycentre, optical properties, attenuation factor and roughness exponent. We provide the essential components of the shader solution in the following pseudo-algorithm.

1. First pass: Generate texture maps.

(a) Render enamel front positions and normals, enamel back positions and normals, and enamel thickness in the viewing direction.

(b) Scale enamel vertex positions to obtain dentine front and back positions. Render dentine back normals, enamel to dentine position map, and dentine thickness in the viewing direction. 
2. Second pass: Render tooth.

(a) Compute multiple scattering in dentine:

i. Determine pixel sample positions on the enamel surface given the 12-tap sample kernel.

ii. Interpolate enamel thickness for each sample.

iii. Estimate incident radiance for each sample by look-up along the normal direction into a prefiltered environment map [25, 26].

iv. Compute radiance transmitted to each mapped dentine sample position using eqs. 1 and 4 .

v. Evaluate the BSSRDF for each sample position [18].

vi. Sum the contributions and compute the radiance that emerges from the enamel layer using eqs. 1 and 4.

vii. Attenuate the total radiance contribution.

(b) Compute outward single scattering:

i. Look up incident radiance in the (normal) environment map using the reflection of the direction toward the camera.

ii. Compute refracted directions into enamel and dentine, and use eq. 3 to compute the outward scattered radiance.

(c) Compute back-lit transmission:

i. Look up enamel and dentine thicknesses in textures.

ii. Compute direct transmission using eq. 1.

(d) Compute reflection in saliva and enamel.

(e) Tone map total radiance to get colour in rgb.

Since the mouth in many cases has little light inside it, it often makes sense with respect to efficiency to omit back-lit transmission (2.c) and outward single scattering from dentine (2.b, dentine part). For optimization, the diffuse reflectance can be precomputed and stored as a single texture for a number of distances corresponding to the desired texture resolution. The diffuse reflectance texture needs only be generated once for a particular set of parameters. The texture map pass should be optimized using multiple render targets.

\section{Results}

Figure 3 is renderings of an incisor ( $\sim 25 \mathrm{k}$ triangles) and a full set of teeth in the lower jaw ( $\sim 200 \mathrm{k}$ triangles). Both were rendered at 20-30 frames per second on an NVIDIA GTX 580 without using the optimizations mentioned at the end of the previous section. Our method is not geometry bound, so the frame rate is similar whether we render a single tooth or a full set of teeth.

Figure 4 allows a qualitative comparison of a crown photographed from two different camera angles and a rendered molar tooth. The molar tooth does not have the same geometry as the crown. The crown is quite realistic (as it should be) with its variations in colour. This is a result of the artistic handwork associated with its production. The renderings only exhibit subtle colour variations which are mostly a result of reflections from the environment map. The attenuation of light scattered from the dentine layer produces a semi-transparent effect 

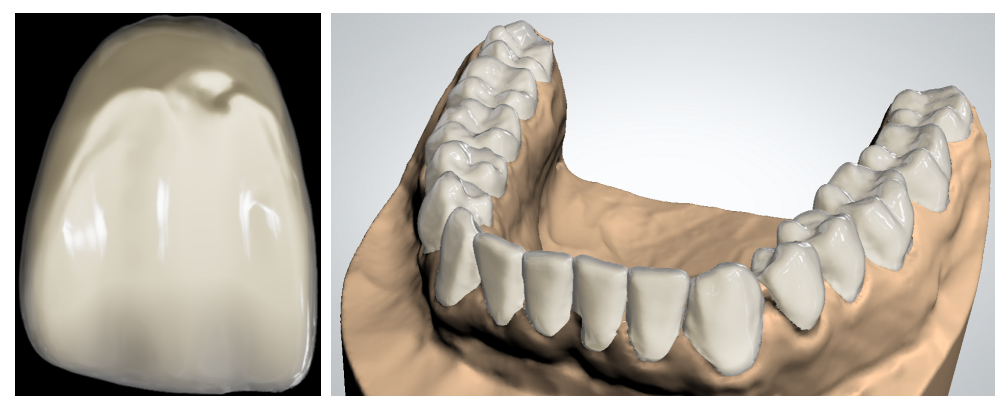

Fig. 3. An incisor (left) and a full set of teeth in the lower jaw (right). Both rendered at 20-30 frames per second.
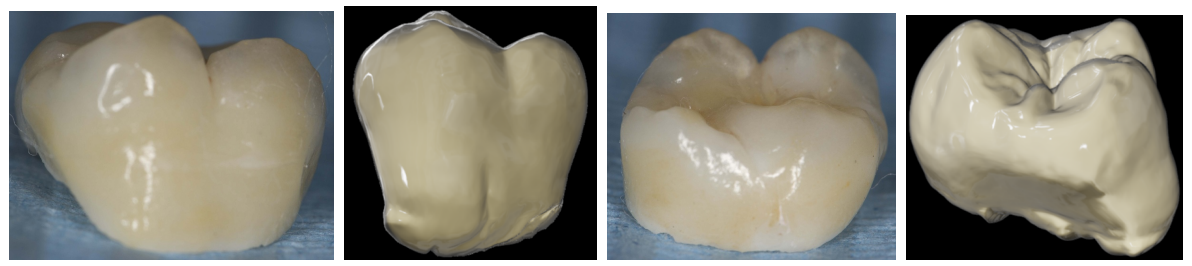

Fig. 4. A molar crown from two different angles compared to renderings of a molar model. The model geometry is not based on the crown.

near the edges, and a slight blueish hue (opalescence) appears due to backscattering in the enamel layer.

In figure 5, we compare two incisor crowns to an incisor model. The incisor comparison is not as convincing as the molar comparison. We believe the reason is a larger deviation between crown geometry and rendered geometry. The dent near the top of the rendered tooth is a damaged area of the tooth. It actually illustrates the softness in illumination that is a result of the 12-tap sampling. The difference in shade between the top and bottom of the rendered tooth is because of variation in the illumination incident from the environment map. Another primary reason for the qualitative differences between crown photos and rendered images is that our appearance model does not include age-related deterioration and discolouring effects (due to smoking, for example).

The techniques that we use for subsurface scattering provide direct control over the material appearance. We use measured optical properties (table 1) as a guide to set reasonable values for absorption and scattering in enamel and dentine. By varying the properties within the allowed margins, we are able to vary how light interacts with the two layers. As illustrated in figure 6, this leads to apparent differences in the appearance of the rendered tooth.

Using our simple interpolation scheme (section 2.3), the enamel thickness is controlled by the parameters $t_{\min }$ and $t_{\max }$. As illustrated in figure 7 , the enamel thickness directly affects the tooth appearance. 

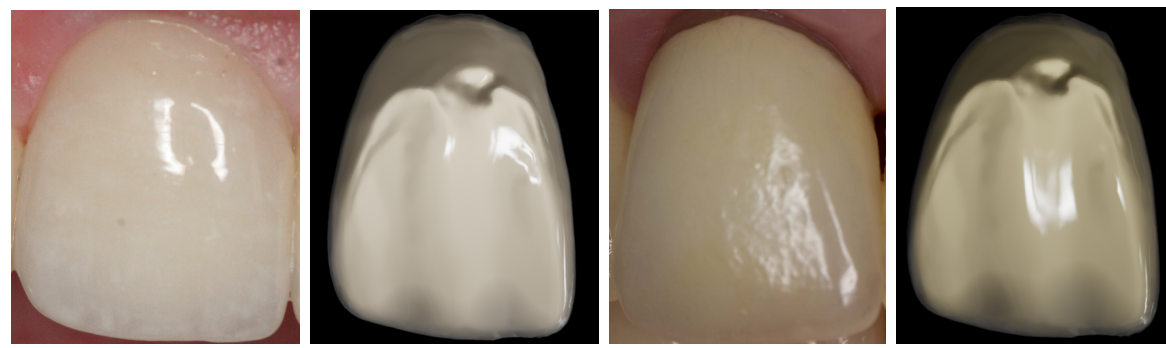

Fig. 5. Two incisor crowns compared to the incisor model rendered with two different sets of parameters.
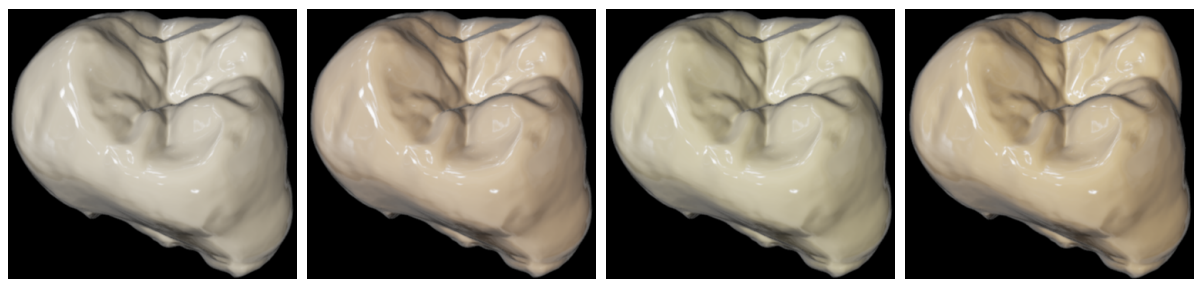

Fig. 6. Variations in colour due to variation in scattering properties. Enamel is changed in the second image, dentine is changed in the third, and both are changed in the fourth.
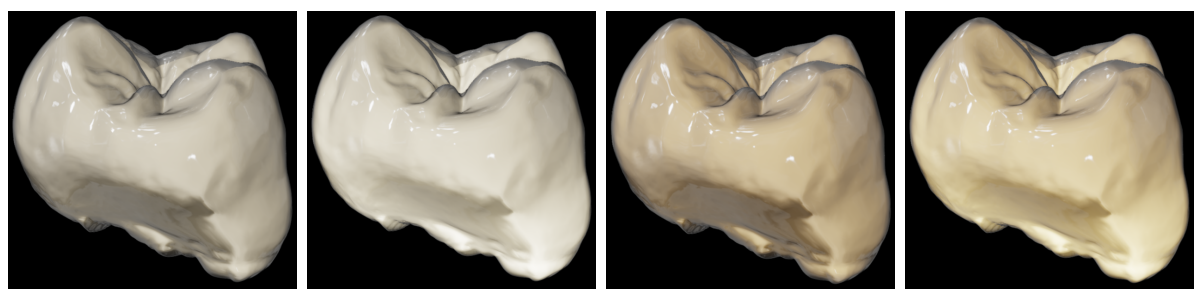

Fig. 7. The effect of enamel thickness. More variation between top and bottom thickness in the second image, more scattering in the third, both in the fourth.

\section{Discussion}

We have presented what we believe is the first real-time rendering technique for teeth that is based on the optical properties of teeth and does not require artistic or computational preprocessing. In this final section, we discuss options for improvement.

The enamel layer scatters light more than what can be captured by single scattering and too little to fulfil the assumption of being a highly scattering material as is required by the diffusion theory. A good real-time method for handling this type of material is left for future work.

The enamel thickness interpolation scheme could be improved. As mentioned in section 1.1, it is possible to acquire the surface of the dentine layer from a CT 
scan. Analysis of a library of such CT scans could lead to an empirically based procedure for estimating enamel thicknesses. We believe that such a procedure could greatly enhance the realism of the renderings that our method can produce.

Teeth have a fairly complex geometry, and the surfaces of enamel and dentine are often curved and dented, especially near the edges. The dipole approximation works under the assumption of a semi-infinite medium with a planar surface. This means that sampling must be (very) local, or alternatively that the assumption must be ignored. Since a dentist should be allowed to manipulate the geometry (the tooth shape) interactively, we must keep the constraint that no precomputation is allowed. Development of an interactive method for subsurface scattering that, without use of precomputation, more accurately handles geometry with sharper features is also left for future work.

It would be an advantage to have more detailed measurements of optical properties for dentine and enamel. The references we have identified do not seem to agree on how to measure these properties nor on the range that they should be in. This leaves room for further study on measuring the optical properties of enamel and dentine. Ideally, such a study would categorise properties by subject, such that it would be easier to analyse and render sets of teeth where parameters such as sex, race, and age are visually reflected.

The proposed model does not handle visual effects related to the natural wear and tear of teeth, such as caries or prolonged exposure to coffee, red wine or cigarettes. Such effects can be introduced through texture mapping. Textures could be generated using an image-based approach (discussed in section 1.1) or automatically using procedural or statistical methods. To apply such textures properly, a study of automatic UV mapping of teeth would also be of interest.

Acknowledgement. This work was partly financed by the Digital Prototypes project which is funded by the Danish Council for Technology and Innovation.

\section{References}

1. Alexander, O., Rogers, M., Lambeth, W., Chiang, J.Y., Ma, W.C., Wang, C.C., Debevec, P.: The digital Emily project: Achieving a photorealistic digital actor. IEEE Computer Graphics and Applications 30 (2010) 20-31

2. Alexander, O., Rogers, M., Lambeth, W., Chiang, M., Debevec, P.: The digital Emily project: Photoreal facial modeling and animation. ACM SIGGRAPH 2009 Course Notes (2009)

3. van Noort, R.: The future of dental devices is digital. Dental Materials 28 (2012) $3-12$

4. Hanrahan, P., Krueger, W.: Reflection from layered surfaces due to subsurface scattering. Proceedings of ACM SIGGRAPH 93 (1993) 165-174

5. Hable, J., Borshukov, G., Hejl, J.: Fast skin shading. In Engel, W., ed.: ShaderX ${ }^{7}$. Charles River Media (2009) 161-173

6. Shetty, S., Bailey, M.: A physical rendering model for human teeth. ACM SIGGRAPH 2010 Posters (2010)

7. Shetty, S.: Layered rendering model for human teeth. Master's thesis, Oregon State University (2011) 
8. Botha, C.P., Post, F.H.: New technique for transfer function specification in direct volume rendering using real-time visual feedback. In Mun, S.K., ed.: Proceedings of the SPIE International Symposium on Medical Imaging 2002: Visualization, Image-Guided Procedures, and Display. Volume 4681. (2002) 349-356

9. Kniss, J., Kindlmann, G., Hansen, C.: Multidimensional transfer functions for interactive volume rendering. IEEE Transactions on Visualization and Computer Graphics 8 (2002) 270-285

10. Kim, L., Park, S.H.: Haptic interaction and volume modeling techniques for realistic dental simulation. The Visual Computer 22 (2006) 90-98

11. Rhienmora, P., Gajananan, K., Haddawy, P., Dailey, M.N., Suebnukarn, S.: Augmented reality haptics system for dental surgical skills training. In: Proceedings of the 17th Symposium on Virtual Reality Software and Technology (VRST '10). (2010) 97-98

12. Yau, H.T., Hsu, C.Y.: Development of a dental training system based on pointbased models. Computer-Aided Design and Applications 3 (2006) 779-787

13. Wang, D.X., Zhang, Y., Wang, Y., Lu, P., Wang, Y.: Development of dental training system with haptic display. In: Proceedings of the 2003 IEEE International Workshop on Robot and Human Interactive Communication. (2003) 159-164

14. Wang, D., Zhang, Y., Wang, Y., Lü, P., Zhou, R., Zhou, W.: Haptic rendering for dental training system. Science in China Series F: Information Sciences 52 (2009) $529-546$

15. Pighin, F., Szeliski, R., Salesin, D.H.: Modeling and animating realistic faces from images. International Journal of Computer Vision 50 (2002) 143-169

16. Zhang, Y., Sim, T., Tan, C.L., Sung, E.: Anatomy-based face reconstruction for animation using multi-layer deformation. Journal of Visual Languages and Computing 17 (2006) 126-160

17. Ishimaru, A.: Wave Propagation and Scattering in Random Media. Academic Press (1978)

18. Jensen, H.W., Marschner, S.R., Levoy, M., Hanrahan, P.: A practical model for subsurface light transport. In: Proceedings of ACM SIGGRAPH 2001. (2001) 511-518

19. Spitzer, D., Bosch, J.T.: The absorption and scattering of light in bovine and human dental enamel. Calcified Tissue Research 17 (1975) 129-137

20. Zijp, J., ten Bosch, J., Groenhuis, R.: HeNe-laser light scattering by human dental enamel. Journal of Dental Research 74 (1995) 1891-1898

21. Fried, D., Glena, R.E., Featherstone, J.D.B., Seka, W.: Nature of light scattering in dental enamel and dentin at visible and near-infrared wavelengths. Applied Optics 34 (1995) 1278-1285

22. Zijp, J.R.: Optical properties of dental hard tissues. Doctoral dissertation, University of Groningen (2001)

23. Henyey, L.G., Greenstein, J.L.: Diffuse radiation in the galaxy. Annales d'Astrophysique 3 (1940) 117-137

24. Jensen, H.W., Legakis, J., Dorsey, J.: Rendering of wet materials. In Lischinski, D., Larson, G.W., eds.: Rendering Techniques '99 (Proceedings of EGWR 1999), Springer (1999) 273-282

25. Miller, G.S., Hoffman, C.R.: Illumination and reflection maps: Simulated objects in real environments. ACM SIGGRAPH 84 Course Notes for Advanced Computer Graphics Animation (1984)

26. Greene, N.: Environment mapping and other applications of world projections. IEEE Computer Graphics and Applications 6 (1986) 21-29 